\section{OPEN ACCESS}

Edited by:

Mark Blenner,

Clemson University, United States

Reviewed by:

Xiaofeng Yang,

South China University of Technology,

China

John Andrew Jones,

Miami University, United States

*Correspondence:

Irina Borodina

irbo@biosustain.dtu.dk

${ }^{\dagger}$ These authors have contributed equally to this work

Specialty section:

This article was submitted to

Synthetic Biology,

a section of the journal

Frontiers in Bioengineering and

Biotechnology

Received: 02 April 2020

Accepted: 22 July 2020

Published: 14 August 2020

Citation:

Arnesen JA, Kildegaard KR, Cernuda Pastor M, Jayachandran S, Kristensen M and Borodina I (2020) Yarrowia lipolytica Strains Engineered for the Production of Terpenoids. Front. Bioeng. Biotechnol. 8:945. doi: 10.3389/fbioe.2020.00945

\title{
Yarrowia lipolytica Strains Engineered for the Production of Terpenoids
}

\section{Jonathan Asmund Arnesen ${ }^{\dagger}$, Kanchana Rueksomtawin Kildegaard ${ }^{+}$, Marc Cernuda Pastor, Sidharth Jayachandran, Mette Kristensen and Irina Borodina*}

The Novo Nordisk Foundation Center for Biosustainability, Technical University of Denmark, Kongens Lyngby, Denmark

Terpenoids are a diverse group of over 55,000 compounds with potential applications as advanced fuels, bulk and fine chemicals, pharmaceutical ingredients, agricultural chemicals, etc. To facilitate their bio-based production, there is a need for plug-andplay hosts, capable of high-level production of different terpenoids. Here we engineer Yarrowia lipolytica platform strains for the overproduction of mono-, sesqui-, di-, tri-, and tetraterpenoids. The monoterpene platform strain was evaluated by expressing Perilla frutescens limonene synthase, which resulted in limonene titer of $35.9 \mathrm{mg} / \mathrm{L}$ and was 100-fold higher than when the same enzyme was expressed in the strain without mevalonate pathway improvement. Expression of Callitropsis nootkatensis valencene synthase in the sesquiterpene platform strain resulted in $113.9 \mathrm{mg} / \mathrm{L}$ valencene, an 8.4-fold increase over the control strain. Platform strains for production of squalene, complex triterpenes, or diterpenes and carotenoids were also constructed and resulted in the production of $402.4 \mathrm{mg} / \mathrm{L}$ squalene, $22 \mathrm{mg} / \mathrm{L}$ 2,3-oxidosqualene, or $164 \mathrm{mg} / \mathrm{L}$ $\beta$-carotene, respectively. The presented terpenoid platform strains can facilitate the evaluation of terpenoid biosynthetic pathways and are a convenient starting point for constructing efficient cell factories for the production of various terpenoids. The platform strains and exemplary terpenoid strains can be obtained from Euroscarf.

Keywords: terpenes, yeast, metabolic engineering, mevalonate pathway, isoprenoids

\section{INTRODUCTION}

Terpenoids comprise the largest class of secondary metabolites; many have biological activity and are used as nutra- and pharmaceutical agents and ingredients for cosmetics or food (Tetali, 2018). Several terpenoids, such as farnesene and bisabolene, have been developed as advanced biofuels. Terpenoids are categorized depending on the number of carbon atoms forming the core skeleton. The commonly studied classes are monoterpenoids $\left(\mathrm{C}_{10}\right)$, sesquiterpenoids $\left(\mathrm{C}_{15}\right)$, diterpenoids $\left(\mathrm{C}_{20}\right)$, triterpenoids $\left(\mathrm{C}_{30}\right)$, and tetraterpenoids $\left(\mathrm{C}_{40}\right)$ also known as carotenoids (Ashour et al., 2010). Other terpenoid classes include hemiterpenoids $\left(C_{5}\right)$ and sesterterpenoids $\left(\mathrm{C}_{25}\right)$ (Janocha et al., 2015). The core hydrocarbon skeletons assembled from isoprene units are commonly modified by enzymes like cytochromes P450, hydrogenases, methyltransferases, and glycosyltransferases. Terpenoids content in natural sources is typically low and extraction 
may result in by-products. As an example, the sweet wormwood Artemisia annua contains artemisinin, a terpene with antimalarial properties, but the content is only $0.8 \%$ of the plant dry weight (Zyad et al., 2018). Similarly, to extract $1 \mathrm{~kg}$ of the flavoring and fragrance ingredient valencene, some sources estimate that 2.5 million $\mathrm{kg}$ of oranges is required (Evolva, 2020 Our valencene the natural choice for flavor and fragrance applications). Some terpenoids can be synthesized chemically, but these processes may be extremely complicated. For example, after two decades of research, a full chemical synthesis of azadirachtin, a triterpenoid with potent insect antifeedant properties, was performed with a yield of $0.00015 \%$ and no less than 71 steps (Fernandes et al., 2019). Therefore, production of complex terpenoids by chemical synthesis may be too expensive to be economically feasible (Zhang et al., 2017a). Production of terpenoids by fermentation using engineered cell factories can be cheaper and more sustainable than chemical synthesis. Indeed, hosts like the yeast Saccharomyces cerevisiae have been engineered to produce a variety of terpenoids and some of these cell factories are currently used industrially (Moser and Pichler, 2019). For example, the sesquiterpene $\beta$-farnesene is being produced industrially by highly engineered $S$. cerevisiae with titers of $130 \mathrm{~g} / \mathrm{L}$ reported in the literature (Meadows et al., 2016). However, since these highly productive, industrial yeast chassis are proprietary, there is a need for accessible microbial terpenoid platform strains. Recently the oleaginous yeast Yarrowia lipolytica has attracted attention as a promising host for the production of hydrophobic compounds. The genome of Y. lipolytica has been sequenced and convenient toolkits for genetic engineering exists (Dujon et al., 2004; Christen and Sauer, 2011; Holkenbrink et al., 2018; Turck et al., 2019). Furthermore, several Y. lipolytica strains have been granted GRAS-status (Groenewald et al., 2014). The species naturally overproduces lipids and hence has a high acetyl-coenzyme A (CoA) flux, which makes it useful for terpenoid production. Terpenoids like limonene, linalool, $\alpha$-farnesene, betulinic acid, and $\beta$-carotene have previously been produced in recombinant Y. lipolytica strains (Yang et al., 2016; Cao et al., 2017; Gao et al., 2017; Cheng et al., 2019; Jin et al., 2019). In yeasts, the $C_{5}$-precursor of terpenoids isopentyl diphosphate (IPP), is produced via the cytosolic mevalonate (MVA) pathway (Figure 1; Ashour et al., 2010; Cao et al., 2016). The initial steps of the MVA-pathway start with the condensation of three acetyl-CoA molecules forming 3-hydroxy3-methylglutaryl-CoA (HMG-CoA). Subsequently, HMG-CoA is reduced to mevalonic acid, which is then phosphorylated twice and lastly decarboxylated, forming IPP which can isomerize to form dimethylallyl diphosphate (DMAPP). These phosphorylated $\mathrm{C}_{5}$-precursors can condensate to form geranyl diphosphate (GPP), farnesyl diphosphate (FPP), geranylgeranyl diphosphate (GGPP) that then generate the backbones of monoterpenoids, sesqui- and triterpenoids, or diterpenoids and carotenoids, respectively. Although Y. lipolytica may be highly suited for the production of terpenoids, the yeast strains often require several cycles of engineering and optimization before high terpenoid titers can be reached. Since terpenoids are derived from the same basic IPP/DMAPP-building blocks, preengineered "platform" strains with high MVA-pathway flux could be engineered (Cravens et al., 2019). Upon integration of terpenoid biosynthetic genes, such platform strain could provide immediately improved titers, which would shorten the downstream engineering process considerably. Furthermore, platform strains tailored toward the production of specific terpenoid classes could be constructed, since the subsequent pathways from IPP/DMAPP diverge toward mono-, sesqui-, tri-, or diterpenoids and carotenoids. Therefore, this study aimed to develop pre-engineered platform strains of $Y$. lipolytica with improved production of GPP for monoterpene production, FPP for sesquiterpene production, squalene/2,3-oxidosqualene for triterpene production, or GGPP for diterpenoid and carotenoid production. While these platform strains require additional engineering before industrially relevant titers can be reached, they can serve as convenient plug-and-play hosts for testing new or improved enzymes toward various terpenoids and for testing new metabolic engineering strategies for improving terpenoid production in Y. lipolytica. By making the strains available via Euroscarf, we hope to initiate a scientific community effort of building superior platform strains together.

\section{MATERIALS AND METHODS}

\section{Yeast Strains and Media}

The W29-derived ST6512 (MatA ku70 4 :PrTEF1->Cas9TTef12:PrGPD->DsdA-TLip2) expressing Cas9 for CRISPR/ based DNA integration was used to construct the platform strains (Marella et al., 2019). This strain was based on the W29 Y. lipolytica (MatA) strain Y-63746, which was a kind gift from the ARS Culture Collection, NCAUR, United States. YPD-media containing $10 \mathrm{~g} / \mathrm{L}$ yeast extract, $20 \mathrm{~g} / \mathrm{L}$ peptone, and $20 \mathrm{~g} / \mathrm{L}$ glucose were used to grow the $Y$. lipolytica strains at $30^{\circ} \mathrm{C}$. $20 \mathrm{~g} / \mathrm{L}$ agar was added for solid media. For selection, either nourseothricin $(250 \mathrm{mg} / \mathrm{L})$ or hygromycin $(400 \mathrm{mg} / \mathrm{L})$ was added to the media. Cultivation of strains for terpenoid production was done in YP-media with $80 \mathrm{~g} / \mathrm{L}$ glucose. Escherichia coli strain $\mathrm{DH} 5 \alpha$ was used for plasmid construction. The E. coli cells were cultivated at $37^{\circ} \mathrm{C}$ on Lysogeny Broth (LB) medium supplemented with $100 \mathrm{mg} / \mathrm{L}$ ampicillin for plasmid selection. The chemicals were obtained, if not indicated otherwise, from Sigma-Aldrich. Nourseothricin was purchased from Jena BioScience GmbH (Germany).

\section{DNA Constructs}

The primers, biobricks, plasmids, and primers used in this study are listed in Supplementary Tables 1-4, respectively. The biobricks were amplified with PCR using Phusion U polymerase (Thermo Scientific) and assembled into the EasyCloneYALI vectors with USER cloning (Holkenbrink et al., 2018). The USER reactions were transformed into E. coli and correct assemblies were verified by sequencing. Codonoptimized genes encoding Citrus limon limonene synthase (ClLS) (Accession: Q8L5K3.1), Perilla frutescens limonene synthase (PfLS) (Accession: AJW68081.1), Artemisia annua $\beta$-farnesene synthase (AaBFS) (MT276895.1), Callitropsis nootkatensis valencene synthase (CnVS) (Accession: AFN21429.1), 


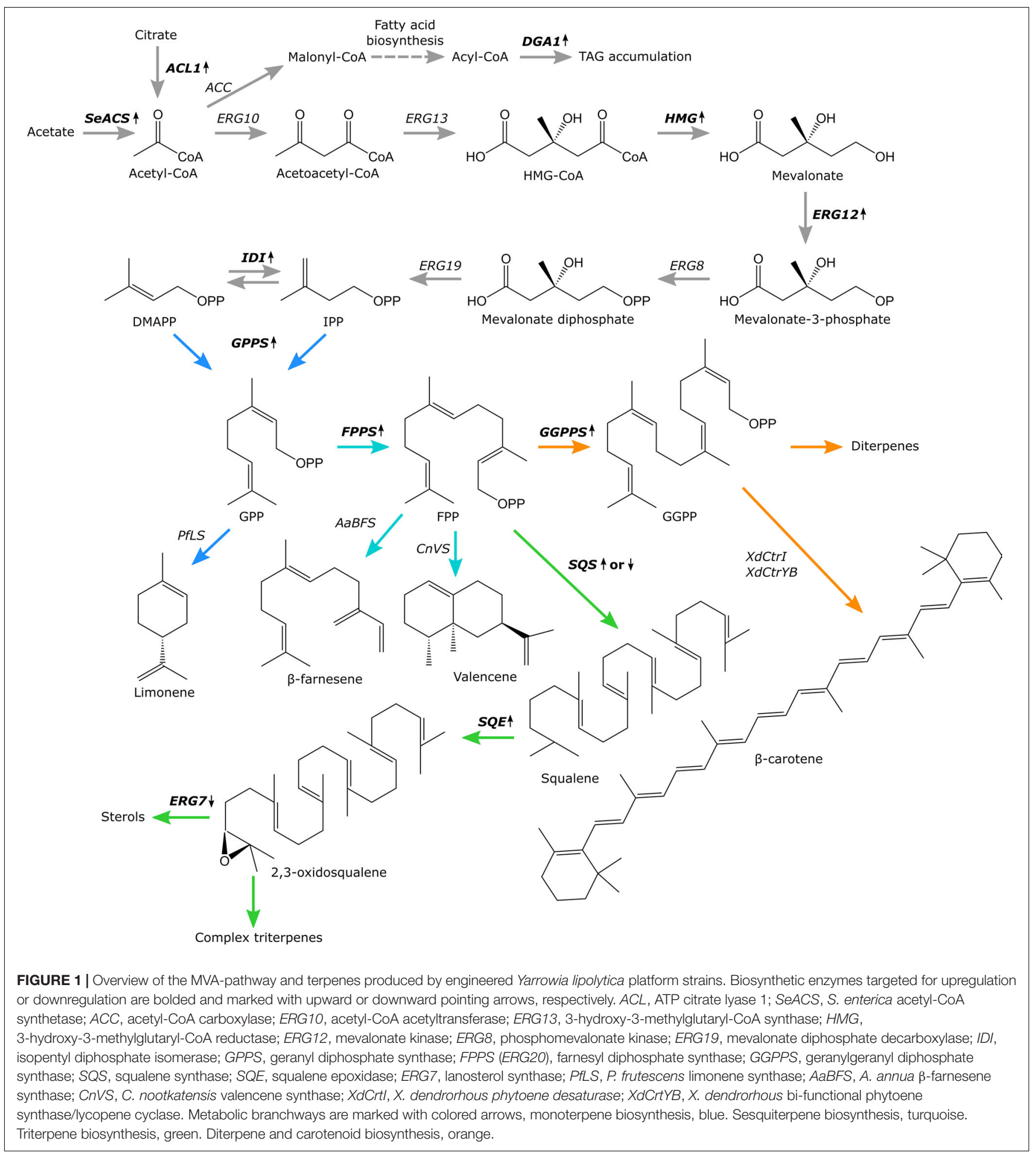

Xanthophyllomyces dendrorhous phytoene desaturase (XdCrtI) (Accession: ATB19150.1), X. dendrorhous bi-functional phytoene synthase/lycopene cyclase (XdCrtYB) (Accession: Q7Z859.1) with an A190T substitution, and Salmonella enterica acetyl-CoA synthetase (SeACS) (Accession: WP_000083882.1) with a L641P substitution as described in Huang et al. (2018) were ordered as
GeneArt String DNA fragments from Thermo Fischer Scientific. The codon-optimized nucleotide sequences can be found the Supplementary Material.

$E R G 20$ was mutated into $E R G 20^{F 88 C}$ based on an alignment of Y. lipolytica Erg20p (Accession number: XP_503599.1) and S. cerevisiae Erg20p (Accession number: CAA89462.1) amino 
sequences (Supplementary Figure 1). The mutated residue was selected based on a previous report (Ignea et al., 2015). The alignment was made using Benchling software and was visualized with MView 1.63 (Benchling, 2020 Cloud-Based Informatics Platform for Life Sciences R\&D | Benchling; Brown et al., 1998). In addition, ERG20 was mutated into ERG20 ${ }^{F 88 ~ W-N 119 W}$ based on a previous report (Cao et al., 2017). The amino acid substitutions were constructed with PCR mutagenesis and the primers are listed in Supplementary Table 4.

\section{Yeast Transformation}

The yeast strains used in this study are listed in Supplementary Table 1. A lithium-acetate based transformation protocol as described previously was used for yeast strain engineering and integration vectors were linearized with NotI-enzyme before transformation (Holkenbrink et al., 2018). Correct integration was verified with colony PCR using vector-specific primers and primers complementary to the genomic region adjacent to the integration site.

\section{Yeast Cultivation}

Yeast strains were inoculated into $2.5 \mathrm{~mL}$ YPD in 24-well plates with air-penetrable lid (EnzyScreen, Netherlands) and grown for $16-24 \mathrm{~h}$ at $30^{\circ} \mathrm{C}$ and $300 \mathrm{rpm}$ agitation at $5 \mathrm{~cm}$ orbit cast. Then for limonene, $\beta$-farnesene, and valencene, the required volume for a starting $600 \mathrm{~nm}$ optical density (OD) of 0.1 was transferred to $2 \mathrm{~mL}$ of YP with $80 \mathrm{~g} / \mathrm{L}$ glucose in glass tubes and a dodecane overlay of $200 \mu \mathrm{L}$ was added. For squalene, 2,3oxidosqualene, and $\beta$-carotene, the required volume for a starting $600 \mathrm{~nm}$ optical density (OD) of 0.1 was transferred to $2.5 \mathrm{~mL}$ of YP with $80 \mathrm{~g} / \mathrm{L}$ glucose in a 24-deepwell plate. The inoculated media was incubated for $72 \mathrm{~h}$ at $30^{\circ} \mathrm{C}$ with $300 \mathrm{rpm}$ agitation. All cultivations were performed in biological triplicates. OD and cell dry weight was measured by the end of cultivation.

\section{Metabolite Analysis}

For limonene, $\beta$-farnesene, and valencene, the cell culture broth was centrifuged and the dodecane phase was harvested for analysis. The dodecane samples were diluted in cyclohexane with either patchoulol or myrcene as an internal standard for valencene and $\beta$-farnesene, or limonene, respectively. $\beta$-carotene, was extracted as previously described (Kildegaard et al., 2017). For squalene and 2,3-oxidosqualene, $1 \mathrm{~mL}$ of culture broth was transferred into a $2 \mathrm{~mL}$ microtube, centrifuged and the supernatant was discarded. $500 \mu \mathrm{L}$ of $0.5-0.75 \mathrm{~mm}$ acid-washed glass beads and $1 \mathrm{~mL}$ of acetonitrile were added to each sample. The samples were then incubated at $95^{\circ} \mathrm{C}$ at $650 \mathrm{rpm}$ shaking for $1 \mathrm{~h}$ in a Thermo-Shaker TS-100 (biosan). Subsequently, the cells were broken with a Precellys ${ }^{\circledR} 24$ homogenizer (Bertin Corp.) four times at $5500 \mathrm{rpm}$ for $20 \mathrm{~s}$ with samples being kept on ice in between rounds of breaking. Lastly, the samples were centrifuged, and the supernatant was collected for analysis.

Gas chromatographic (GC) analysis for valencene, limonene, and $\beta$-farnesene was conducted using Thermo Scientific GC Trace 1300 equipment with a flame ionization detector (FID) and equipped with a fused-silica capillary column (BPX5, $30 \mathrm{~m} \times 0.25 \mathrm{~mm}$ ID, $0.25 \mu \mathrm{m}$, SGE Analytical Science). Helium at a constant flow rate of $1.0 \mathrm{~mL} / \mathrm{min}$ was used as the carrier gas. The GC oven temperature started at $50^{\circ} \mathrm{C}$ for $1.5 \mathrm{~min}$ and then increased to $170^{\circ} \mathrm{C}$ at $30^{\circ} \mathrm{C} / \mathrm{min}$ and hold for $1.5 \mathrm{~min}$. Then from 170 to $300^{\circ} \mathrm{C}$ at $15^{\circ} \mathrm{C} / \mathrm{min}$ and hold for $4.5 \mathrm{~min}$, finalizing the chromatographic run at $20.2 \mathrm{~min}$. The injector and detector ports were both kept at $300^{\circ} \mathrm{C}$ and the injector operated in a split mode of 20:1. Concentrations of beta-carotene, squalene, and 2,3-oxidosqualene were measured using a Dionex Ultimate 3000 HPLC with a Supelco Discovery HS F5-3 HPLC column $(150 \times 2.1 \mathrm{~mm}, 3 \mu \mathrm{m}$ particle size $)$ and a DAD-3000 Diode Array Detector at 450 and $210 \mathrm{~nm}$, respectively. The mobile phase consisted of A: $10 \mathrm{mM}$ ammonium formate and B: acetonitrile. The flow rate was $0.7 \mathrm{~mL} / \mathrm{min}$ and the column was kept at $30^{\circ} \mathrm{C}$. The mobile phase was introduced as $25 \% \mathrm{~B}$ and held at this composition for $2 \mathrm{~min}$. The gradient was then ramped to $90 \% \mathrm{~B}$ to $4 \mathrm{~min}$ and this gradient held to $10.5 \mathrm{~min}$ and followed by a linear gradient to $25 \% \mathrm{~B}$ at $11.25 \mathrm{~min}$. The column was equilibrated with $25 \%$ B until $13.5 \mathrm{~min}$. Samples were held at $5^{\circ} \mathrm{C}$ during the analysis and $1 \mu \mathrm{L}$ sample volume injected. Data analysis was performed using Chromeleon 7.2.9 and the analyte quantification was performed using peak area ratios of authentic standards. Quantities of limonene for ST9249, ST9395, and squalene for ST6512 were extrapolated from the standard curve regression.

\section{RESULTS}

\section{Design of Y. lipolytica Platform Strains for Terpenoid Production}

To design the platform strains, we compiled a list of studies for terpenoid production in Y. lipolytica (Table 1). While some studies generated highly modified strains by, for example, overexpressing the entire MVA-pathway, it may not be clear from the experimental design, which exact modifications had resulted in improvement of terpenoid titers. Therefore, we selected metabolic engineering strategies that had been clearly demonstrated to result in improved terpenoid production. These strategies formed the basis of the platform strains. Two key strategies were implemented for all platform strains: improvement of the precursor acetyl-CoA pool and upregulation of the MVA-pathway to improve the accumulation of IPP/DMAPP (Figure 1). Increasing the acetyl-CoA pool was done by overexpression of the native ATP citrate lyase 1 (ACL) and the Salmonella enterica acetyl-CoA synthetase (SeACS), as this was shown to increase squalene titers in Y. lipolytica in combination with overexpression of the native 3-hydroxy-3methylglutaryl-CoA reductase $(H M G)$ (Huang et al., 2018). In Y. lipolytica, Aclp generates acetyl-CoA and oxaloacetate from citrate, whereas SeAcsp produces acetyl-CoA from acetate and CoA. To increase MVA-pathway flux, several genes involved in this pathway were overexpressed. The formation of mevalonic acid from 3-hydroxy-3-methylglutaryl-CoA is catalyzed by Hmgp and this is considered a key limiting step in the MVApathway (Ashour et al., 2010). Overexpression of $H M G$ has been shown to boost the production of $\alpha$-farnesene, linalool and limonene in Y. lipolytica (Cao et al., 2016, 2017; Yang et al., 2016). Although truncated versions of Hmgp have been used in S. cerevisiae, studies indicate that the non-truncated version is superior for terpenoid production in Y. lipolytica (Cao et al., 2016; 
TABLE 1 | Metabolic engineering of Yarrowia lipolytica for terpenoid biosynthesis.

\begin{tabular}{|c|c|c|c|c|c|}
\hline Compound & $\begin{array}{l}\text { Carbon } \\
\text { Source }\end{array}$ & $\begin{array}{l}\text { Parental } \\
\text { Strain }\end{array}$ & $\begin{array}{l}\text { Relevant modifications related to } \\
\text { terpenoid biosynthesis }\end{array}$ & Titer & References \\
\hline \multicolumn{6}{|l|}{ Monoterpenoids } \\
\hline \multirow[t]{4}{*}{ Limonene } & Glucose & Po1g & $\uparrow \boldsymbol{H M G}(\uparrow \boldsymbol{C I L S}$ or $\uparrow M S L S)$ & $\begin{array}{l}\text { D-limonene: } 11.7 \mathrm{mg} / \mathrm{L} \\
\text { L-limonene: } 11.1 \mathrm{mg} / \mathrm{L} \\
\text { (bioreactor) }\end{array}$ & Pang et al., 2019 \\
\hline & $\begin{array}{l}\text { Glycerol } \\
\text { Citrate }\end{array}$ & Po1f & $\uparrow A r t L S \uparrow S / t N D P S 1 \uparrow \mathbf{H M G 1} \uparrow \mathbf{E R G 1 2}$ & 165.3 mg/L (bioreactor) & Cheng et al., 2019 \\
\hline & $\begin{array}{c}\text { Glucose } \\
\text { Pyruvic acid }\end{array}$ & Po1f & $\uparrow A r t L S \uparrow S / t N D P S 1 \uparrow \mathbf{H M G 1} \uparrow \mathbf{E R G 1 2}$ & 23.6 mg/L (shake flask) & Cao et al., 2016 \\
\hline & Glucose & ATCC 20460 & $\begin{array}{c}\uparrow H M G 1 \uparrow E R G 12 \uparrow A C L 1 \uparrow S e A C S \uparrow I D I \\
\uparrow E R G 20^{F 88 W-N 119 W} \downarrow S Q S \uparrow P f L S\end{array}$ & 35.9 mg/L (glass tube) & This study \\
\hline Linalool & $\begin{array}{c}\text { Citrate } \\
\text { Pyruvate }\end{array}$ & Po1f & $\uparrow A a L I S \uparrow E R G 20^{F 88 W-N 119 W} \uparrow H M G \uparrow I D I$ & 6.96 mg/L (shake flask) & Cao et al., 2017 \\
\hline \multicolumn{6}{|l|}{ Sesquiterpenoids } \\
\hline \multirow[t]{2}{*}{$\alpha$-farnesene } & $\begin{array}{l}\text { Glucose } \\
\text { Fructose }\end{array}$ & Po1h & $\uparrow S c t H M G \uparrow I D I ~ \uparrow M d F S-L-E R G 20$ & 259.98 mg/L (bioreactor) & Yang et al., 2016 \\
\hline & Glucose & Po1f & $\begin{array}{c}\uparrow B d H M G \uparrow E R G 13 \uparrow M d F S-L-E R G 20 \uparrow E R G 12 \uparrow I D I \\
\uparrow E R G 8,19 \uparrow G P P S\end{array}$ & $25.55 \mathrm{~g} / \mathrm{L}$ (bioreactor) & Liu et al., 2019 \\
\hline$\beta$-farnesene & Glucose & ATCC 20460 & $\begin{array}{c}\uparrow H M G 1 \uparrow E R G 12 \uparrow A C L 1 \uparrow S e A C S \uparrow I D I \uparrow E R G 20 \\
\uparrow A a B F S\end{array}$ & 955 mg/L (glass tube) & This study \\
\hline \multirow[t]{2}{*}{$\beta$-ionone } & Glucose & Po1f & 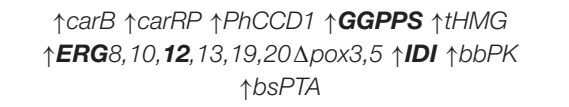 & $0.98 \mathrm{~g} / \mathrm{L}$ (bioreactor) & Lu et al., 2020 \\
\hline & Glucose & Po1f & $\begin{array}{c}\uparrow c a r B \uparrow c a r R P \uparrow O f C C D 1 \uparrow S s N p h T 7 \uparrow H p I D I \\
\uparrow E R G 8,10, \mathbf{1 2}, 13,19 \uparrow t H M G 1 \uparrow \mathbf{G P P S} \\
\uparrow E R G 20 \text {-GGPPS }\end{array}$ & 380 mg/L (bioreactor) & Czajka et al., 2018 \\
\hline$\alpha$-santalene & Glucose & ATCC 201249 & $\uparrow C I S T S \uparrow E R G 8 \uparrow \boldsymbol{H M G}$ & 27.92 mg/L (bioreactor) & Jia et al., 2019 \\
\hline $\begin{array}{l}\text { Nootkatone } \\
\text { valencene }\end{array}$ & Glucose & ATCC 201249 & $\uparrow \mathbf{C n V S} \uparrow C n C Y P 706 M 1-A t A T R 1 \uparrow t H M G \uparrow \mathbf{E R G 2 0}$ & $\begin{array}{l}\text { Nootkatone: } 978.2 \mu \mathrm{g} / \mathrm{L} \\
\text { Valencene: } 22.8 \mathrm{mg} / \mathrm{L} \\
\text { (shake flask) }\end{array}$ & Guo et al., 2018 \\
\hline Valencene & Glucose & ATCC 20460 & $\begin{array}{c}\uparrow H M G 1 \uparrow E R G 12 \uparrow A C L 1 \uparrow S e A C S \uparrow I D I \uparrow E R G 20 \\
\downarrow S Q S \uparrow C n V S\end{array}$ & 113.9 mg/L (glass tube) & This study \\
\hline Amorphadiene & Glucose & Po1g & $\uparrow A a A D S \uparrow \mathbf{H M G 1} \uparrow \mathbf{E R G 1 2}$ & 171.5 mg/L (shake flask) & $\begin{array}{l}\text { Marsafari and Xu, } \\
\qquad 2020\end{array}$ \\
\hline \multicolumn{6}{|l|}{ Triterpenoids } \\
\hline \multirow[t]{2}{*}{ Campesterol } & $\begin{array}{l}\text { Sunflower } \\
\text { seed oil }\end{array}$ & ATCC 201249 & $\triangle E R G 5 \uparrow X I D H C R 7$ & 453 mg/L (bioreactor) & Du et al., 2016 \\
\hline & $\begin{array}{l}\text { Sunflower } \\
\text { seed oil }\end{array}$ & ATCC 201249 & $\triangle E R G 5 \uparrow D r D H C R 7 \uparrow P O X 2$ & 942 mg/L (bioreactor) & Zhang et al., 2017b \\
\hline Ginsenoside K & Glucose & ATCC 201249 & $\begin{array}{c}\uparrow t H M G \uparrow E R G 20 \uparrow S Q S \uparrow P g D S \uparrow P g P P D S \uparrow A t A T R 1 \\
\uparrow P g \cup G T 1\end{array}$ & 161.8 mg/L (bioreactor) & Li et al., 2019 \\
\hline Oleanolic Acid & Glucose & ATCC 201249 & $\begin{array}{c}\uparrow t H M G \uparrow E R G 20 \uparrow S Q S \uparrow G g B A S \\
\uparrow M t C Y P 716 A 12-L-A t A T R 1\end{array}$ & 540.7 mg/L (bioreactor) & Li et al., 2020 \\
\hline \multirow[t]{2}{*}{ Betulinic acid } & Glycerol & ATCC 201249 & $\uparrow t H M G 1 \uparrow S Q S \uparrow A t L U P 1 \uparrow M t C Y P 716 A 12 \uparrow A t A T R 1$ & 26.53 mg/L (shake flask) & Sun et al., 2019 \\
\hline & Glucose & ATCC 201249 & $\begin{array}{c}\uparrow R C L U S \uparrow B P L O \uparrow L j C P R \uparrow S Q S \uparrow S Q E \text { HMG1 } \\
\uparrow M F E 1\end{array}$ & 204.89 mg/L (shake flask) & Jin et al., 2019 \\
\hline \multirow[t]{3}{*}{ Squalene } & $\begin{array}{l}\text { Glucose } \\
\text { Citrate }\end{array}$ & Po1f & $\uparrow H M G 1 \uparrow A C L 1 \uparrow S e A C S$ & 10 mg/gDCW (shake flask) & Huang et al., 2018 \\
\hline & Glucose & $\begin{array}{c}\text { ATCC } \\
\text { MYA2613 }\end{array}$ & 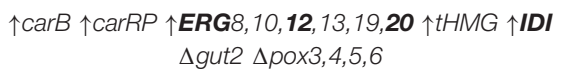 & $531.6 \mathrm{mg} / \mathrm{L}$ & Gao et al., 2017 \\
\hline & Glucose & ATCC 20460 & $\begin{array}{c}\uparrow H M G 1 \uparrow E R G 12 \underset{ }{\downarrow} E R G 7 \uparrow A C L 1 \uparrow S e A C S \uparrow I D I \\
\uparrow E R G 20 \uparrow S Q S\end{array}$ & 402.4 mg/L (deepwell plate) & This study \\
\hline 2,3-oxidosqualene & Glucose & ATCC 20460 & $\begin{array}{c}\uparrow H M G 1 \uparrow E R G 12 \downarrow E R G 7 \uparrow A C L 1 \uparrow S e A C S \uparrow I D I \\
\uparrow E R G 20 \uparrow S Q S \uparrow S Q E\end{array}$ & 22 mg/L (deepwell plate) & This study \\
\hline Protopanaxadiol & Xylose & ATCC 201249 & $\begin{array}{c}\uparrow S s X Y L 1 \uparrow S s X Y L 2 \uparrow X K S \uparrow P g D S \\
\uparrow P g P P D S-L-A t A T R 1 \uparrow t H M G \uparrow E R G 20 \uparrow S Q S \uparrow T K L \\
\uparrow T A L \uparrow T X \Delta p o \times 1,2,3\end{array}$ & 300.63 mg/L (bioreactor) & Wu et al., 2019 \\
\hline
\end{tabular}


TABLE 1 | Continued

\begin{tabular}{|c|c|c|c|c|c|}
\hline Compound & $\begin{array}{l}\text { Carbon } \\
\text { Source }\end{array}$ & $\begin{array}{l}\text { Parental } \\
\text { Strain }\end{array}$ & $\begin{array}{l}\text { Relevant modifications related to } \\
\text { terpenoid biosynthesis }\end{array}$ & Titer & References \\
\hline \multicolumn{6}{|l|}{ Carotenoids } \\
\hline \multirow[t]{2}{*}{ Lycopene } & Glucose & $\mathrm{H} 222$ & $\begin{array}{c}\uparrow P a C r t B \uparrow P a C r t / \uparrow \mathbf{G G P P S} \uparrow \boldsymbol{H M G 1} \Delta p o x 1-6 \\
\Delta \text { gut2 }\end{array}$ & 16 mg/gDCW (bioreactor) & $\begin{array}{l}\text { Matthäus et al., } \\
\qquad 2014\end{array}$ \\
\hline & Glucose & Po1f & $\uparrow \boldsymbol{H} \boldsymbol{M G} \uparrow P a C r t E \uparrow P a C r t B ~ \uparrow P a C r t l \uparrow E R G 19$ & $21.1 \mathrm{mg} / \mathrm{gDCW}$ (bioreactor) & $\begin{array}{l}\text { Schwartz et al., } \\
2017\end{array}$ \\
\hline \multirow[t]{4}{*}{$\beta$-Carotene } & Glucose & $\begin{array}{c}\text { ATCC } \\
\text { MYA2613 }\end{array}$ & $\begin{array}{c}\uparrow c a r B \uparrow c a r R P \uparrow E R G 8,10, \mathbf{1 2}, 13,19,20 \uparrow \mathbf{G G P P S} \\
\uparrow t H M G \uparrow \mathbf{I D I} \Delta \text { Dox3,4,5,6 }\end{array}$ & $4 \mathrm{~g} / \mathrm{L}$ (bioreactor) & Gao et al., 2017 \\
\hline & Glucose & Po1f & $\uparrow \operatorname{carB} \uparrow \operatorname{carRP} \uparrow \mathbf{G G P P S} \uparrow \boldsymbol{H M G} \uparrow E R G 13 \Delta$ pox2,3 & $4.5 \mathrm{~g} / \mathrm{L}$ (bioreactor) & Zhang et al., 2020 \\
\hline & Glucose & ATCC 20460 & $\begin{array}{c}\uparrow \operatorname{carB} \uparrow \operatorname{car} R P \uparrow \boldsymbol{H M G} \uparrow \mathbf{G G P P S} \uparrow D G A 2 \\
\uparrow G P D 1 \Delta p o x 1-6 \Delta \operatorname{tg} / 4\end{array}$ & $6.5 \mathrm{~g} / \mathrm{L}$ (bioreactor) & $\begin{array}{l}\text { Larroude et al., } \\
\qquad 2018\end{array}$ \\
\hline & Glucose & ATCC 20460 & $\begin{array}{c}\uparrow H M G 1 \uparrow E R G 12 \uparrow A C L 1 \uparrow S e A C S \uparrow I D I \\
\uparrow G G P P S \downarrow S Q S \uparrow X d c r t Y B \uparrow X d c r t I\end{array}$ & 164 mg/L (deepwell plate) & This study \\
\hline \multirow[t]{2}{*}{ Astaxanthin } & Glucose & GB20 & $\begin{array}{c}\uparrow \boldsymbol{X d c r t Y B} \uparrow \boldsymbol{X d c r t I} \uparrow \boldsymbol{H M G} \downarrow \mathbf{S Q S} \uparrow X d c r t E \uparrow P s c r t W \\
\uparrow P a c r t Z\end{array}$ & 54.6 mg/L (microtiter plate) & $\begin{array}{l}\text { Kildegaard et al., } \\
\qquad 2017\end{array}$ \\
\hline & Glucose & GB20 & $\begin{array}{c}\uparrow \boldsymbol{X d c r t Y B} \uparrow \boldsymbol{X d c r t I} \uparrow \boldsymbol{H M G}(\uparrow S Q S \uparrow X d c r t E \\
\uparrow S s G G P P S \uparrow H p B K T \uparrow H p C r t Z\end{array}$ & 285 mg/L (bioreactor) & $\begin{array}{l}\text { Tramontin et al., } \\
2019\end{array}$ \\
\hline
\end{tabular}

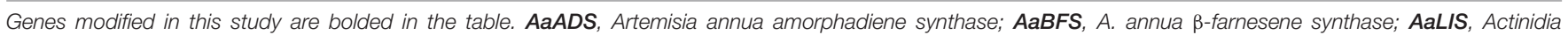

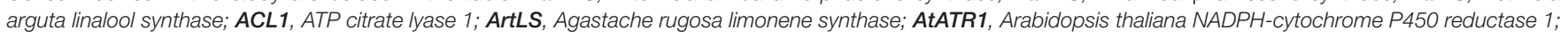

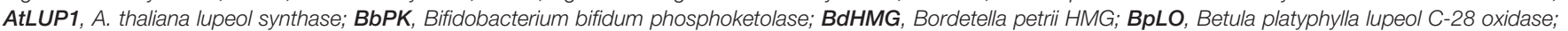

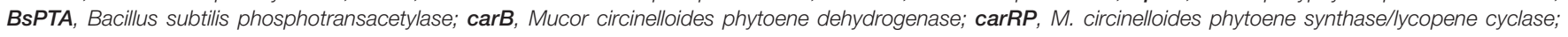

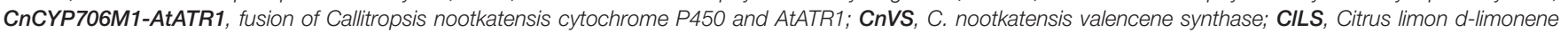

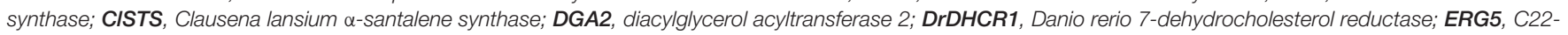
sterol desaturase; ERG7, lanosterol synthase; ERG8, phosphomevalonate kinase gene; ERG10, acetyl-CoA C-acetyltransferase; ERG12, mevalonate kinase; ERG13, Hydroxymethylglutaryl-COA synthase; ERG19, mevalonate diphosphate decarboxylase encoding gene; ERG20, farnesyl diphosphate synthase; ERG20F88W-N119W, geranyl diphosphate synthase; ERG20-GGPPS, fusion of ERG20 and GGPPS, geranylgeranyl diphosphate synthase; GgBAS, Glycyrrhiza glabra $\beta$-amyrin synthase; GPD, glyceraldehyde-3-phosphate dehydrogenase; GPPS, geranyl diphosphate synthase; HMG, Hydroxymethylglutaryl-CoA reductase; HpBKT, Haematococcus pluvialis $\beta$-carotene ketolase; $\mathrm{HpIPI}$, Haematococcus pluvialis isopentyl diphosphate isomerase; $\mathrm{HpCrtZ,} \mathrm{H.} \mathrm{pluvialis} \beta$-carotene hydroxylase; IDI, isopentyl diphosphate

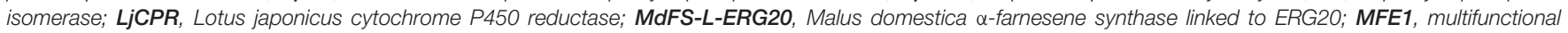
B-oxidation enzyme 1; MsLS, Mentha spicata l-limonene synthase; MtCYP716A12-L-AtATR1, Medicago truncatula cytochrome P450 fused to AtATR1; OfCCD1,

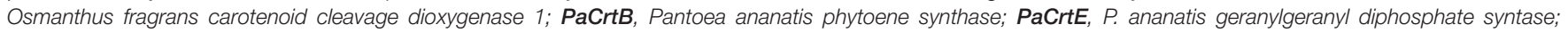

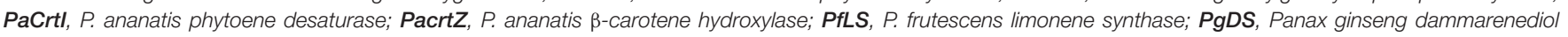
II synthase; PgPPDS, P. ginseng cytochrome P450 enzyme; PgPPDS-L-AtATR1, PgPPDS linked to AtATR1; PgUGT1, P. ginseng UDP-glycosyltransferase; PhCCD1,

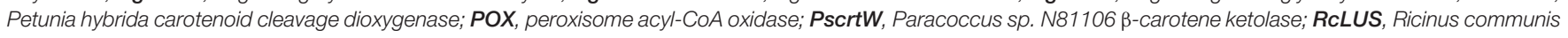

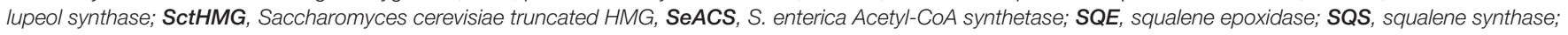

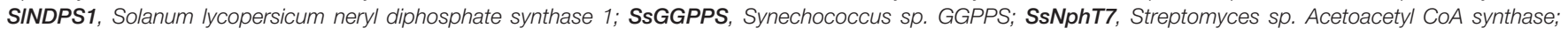

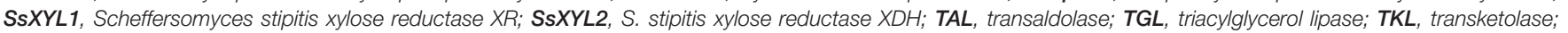

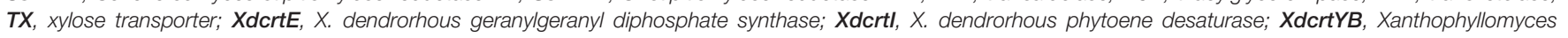
dendrorhous bi-functional phytoene synthase/lycopene cyclase; XKS, xylulose kinase; XIDHCR7, Xenopus laevis 7-dehydrocholesterol reductase.

Kildegaard et al., 2017; Bröker et al., 2018; Han et al., 2018). For example, the production of $\beta$-carotene was enhanced to a greater degree by $H M G$ compared to $t H M G$ both when solely expressed or in combination with the geranylgeranyl diphosphate synthase (GGPPS) (Kildegaard et al., 2017). Indeed, non-truncated HMG has also been used for production of several other terpenoids in Y. lipolytica such as betulinic acid (Table 1; Jin et al., 2019). Furthermore, overexpression of the mevalonate kinase (ERG12) in combination with $H M G$ was shown to increase limonene titers in Y. lipolytica (Cao et al., 2016). Lastly, overexpression of the isopentyl diphosphate isomerase (IDI) that catalyzes the isomerization of IPP and DMAPP together with HMG has been shown to increase the production of linalool and $\alpha$-farnesene in Y. lipolytica (Yang et al., 2016; Cao et al., 2017). Therefore, overexpression of $H M G, E R G 12$, and IDI was selected to form the basis of the terpenoid platform strains. To further tailor the platform strains toward the production of either mono-, sesqui-, tri- or diterpenoids and carotenoids, strategies were implemented to direct IPP/DMAPP toward the appropriate phosphorylated isoprene unit and prevent the flux of this substrate toward undesired products. Monoterpenoids are derived from the GPP which is formed by the condensation of DMAPP and IPP (Ignea et al., 2014). Mutating the phenylalanine residue in position 96 and the asparagine residue in position 127 to tryptophan of $S$. cerevisiae Erg20p changed its function to a geranyl diphosphate synthase (Ignea et al., 2014). A Y. lipolytica Erg20p version with identical mutations in the positionally similar residues (ERG20 $\left.0^{F 8} \mathrm{~W}-\mathrm{N} 119 \mathrm{~W}\right)$ was used for linalool production in Y. lipolytica (Cao et al., 2017). Therefore, the mutated $Y$. lipolytica variant was selected to direct the flux of IPP/DMAPP toward monoterpenoid production. Both sesquiterpenoids and triterpenoids use FPP as the starting substrate, with FPP being dephosphorylated and typically re-arranged to form sesquiterpene backbones, while two units of FPP dimerize to form squalene, which is a precursor for many triterpenoids and sterols. Therefore, the native farnesyl diphosphate synthase (ERG20) was overexpressed in the sesqui- and triterpenoid platform strains, which also had been used to increase nootkatone 
production in Y. lipolytica (Guo et al., 2018). Furthermore, since complex, cyclical triterpenoids often derive from 2,3oxidosqualene, the squalene synthase (SQS) and squalene epoxidase $(S Q E)$ were overexpressed in the triterpenoid platform strain to increase 2,3-oxidosqualene formation. The substrate for diterpenoids and carotenoids is GGPP. Therefore, either the geranylgeranyl diphosphate synthase (GGPPS) or a mutated version of the native Erg20p, where the phenylalanine residue in position 96 was changed to tryptophan $\left(E R G 20^{F 88 C}\right)$, were overexpressed to generate two different diterpene/carotenoid platform strains (Supplementary Figure 1). A similar mutation in the $S$. cerevisiae Erg20p was demonstrated to change its function to a geranylgeranyl diphosphate synthase and improve diterpene production in S. cerevisiae (Ignea et al., 2015). To prevent carbon loss to undesired products, we sought to downregulate squalene formation in the mono-, sesqui-, diterpenoid and carotenoid platform strains by exchanging the native squalene synthase promoter ( $p E R G 9)$ with the relatively weak lanosterol 14- $\alpha$-demethylase promoter ( $p E R G 11$ ). Squalene is essential for sterol production which necessitates downregulation rather than deletion of $S Q S$ and the former strategy had previously been shown to increase $\beta$-carotene production in $Y$. lipolytica (Kildegaard et al., 2017). For the triterpene platform strain, the native lanosterol synthase promoter ( $p E R G 7)$ was truncated to decrease the flux of 2,3oxidosqualene toward sterol synthesis. Both truncations leaving either a 100 or 50 remaining base pairs (bp) of the $3^{\prime}$ end of pERG7 were constructed. By implementing these four strategies, platform strains for either mono-, sesqui-, tri-, or diterpene/carotenoids were constructed.

\section{Evaluation of the Metabolic Engineering Strategies Using $\beta$-Farnesene as the Test Case}

The selected metabolic engineering strategies were evaluated using sesquiterpene $\beta$-farnesene as the test compound. The strategies were then combined to generate platform strains without heterologous terpenoid pathways. $\beta$-farnesene is an acyclic sesquiterpene with potential applications as a biofuel precursor (George et al., 2015). Furthermore, $\beta$-farnesene has been produced at high titers in $S$. cerevisiae, which makes it an excellent compound for testing metabolic engineering strategies (Meadows et al., 2016). Therefore, the $\beta$-farnesene synthase from Artemisia annua (AaBFS) was integrated into the $Y$. lipolytica genome under expression of the pTEFintron promoter. This test-strain solely expressing AaBFS produced $212.7 \pm 5.6 \mathrm{mg} / \mathrm{L} \beta$-farnesene (Figure 2). Subsequently, the strategies for improvement of sesquiterpene production were implemented to discern their effect on $\beta$-farnesene production. The strategies were performed consecutively on each newly generated strain to demonstrate the cumulative effect of the modifications. First, the native $Y$. lipolytica genes $H M G$ and ERG12 were overexpressed in the $\beta$-farnesene producing strain, which increased $\beta$-farnesene titers to $631 \pm 46.1 \mathrm{mg} / \mathrm{L}$, an approximately 3 -fold increase compared to the strain solely expressing AaBFS. Subsequently, overexpression of IDI and
ERG20 resulted in $729 \pm 13.8 \mathrm{mg} / \mathrm{L} \beta$-farnesene. Thirdly, the genes $A C L$ and SeACS were expressed, which raised the titer to $955 \pm 45.1 \mathrm{mg} / \mathrm{L}$, a 4.5 -fold increase compared to the sole expression of $A a B F S$. Lastly, the native squalene promoter was replaced with the weak promoter pERG11 ( $p E R G 11 \_S Q S$ ). However, promoter replacement reduced the $\beta$-farnesene titer to $757 \pm 14.5 \mathrm{mg} / \mathrm{L}$.

\section{Monoterpene Platform Strain}

A platform strain for monoterpene production was constructed by expression of HMG, ERG12, ACL, SeACS, IDI, $E R G 20^{F 88 W-N 119 W}$, and swapping the native squalene promoter (pERG11_SQS). To test the potential of the monoterpene platform strain, either a limonene synthase from Perilla frutescens (PfLS) or Citrus limon (ClLS) was expressed in the platform strain and the base strain. Limonene is a cyclic monoterpene used extensively as a flavor and fragrance ingredient due to its citrus-like characteristics (Cao et al., 2016). No limonene was detected for the base strain expressing ClLS (Figure 3A). The monoterpene platform strain expressing ClLS produced $0.1 \pm 0.2 \mathrm{mg} / \mathrm{L}$ limonene. The base strain expressing PfLS produced $0.36 \pm 0.04 \mathrm{mg} / \mathrm{L}$ limonene, while the monoterpene platform strain expressing PfLS produced $35.9 \pm 1.1 \mathrm{mg} / \mathrm{L}$ limonene. This represents an almost 100 -fold increase in limonene titer compared to the base strain.

\section{Sesquiterpene Platform Strain}

In parallel with the $\beta$-farnesene producing test-strain, a sesquiterpene platform strain was generated with the same modifications except for the integration and expression of AaBFS. This sesquiterpene platform strain was tested by expression of a valencene synthase from Callitropsis nootkatensis (CnVS) generating strain ST9204. Valencene is a cyclic sesquiterpene with a citrus-like aroma used for flavoring or fragrances (Beekwilder et al., 2014). For comparison, CnVS was expressed in a strain without any improvements generating strain ST9396. The sesquiterpene platform strain ST9204 produced $113.9 \pm 6.6 \mathrm{mg} / \mathrm{L}$ valencene, while ST9396 produced $13.5 \pm 2.8 \mathrm{mg} / \mathrm{L}$ (Figure 3B). This corresponds to an 8.4-fold increase in titer and demonstrates the utility of the sesquiterpene platform strain.

\section{Triterpene Platform Strain}

Squalene is a sought-after compound as a food and cosmetics additive, while many complex triterpenoids have pharmaceutical properties (Xu et al., 2016; Hill and Connolly, 2017). Therefore, platform strains for the production of squalene and complex triterpenoids were constructed. This was achieved by consecutively modifying the base strain (ST6512) through several rounds of engineering. Since squalene is naturally produced by $Y$. lipolytica, we could test the production in each intermediate strain constructed toward the final squalene and triterpene platform strain. First, $H M G$ and ERG12 were expressed which resulted in the production of $300.7 \pm 29.1 \mathrm{mg} / \mathrm{L}$ squalene (Figure 4). Secondly, the native lanosterol promoter was truncated to leave either $100 \mathrm{bp}$ or $50 \mathrm{bp}$ of the promoter directly upstream of the lanosterol synthase gene ( $p E R G 7 \_100 b p$ or pERG7_50bp, respectively) generating ST9009 


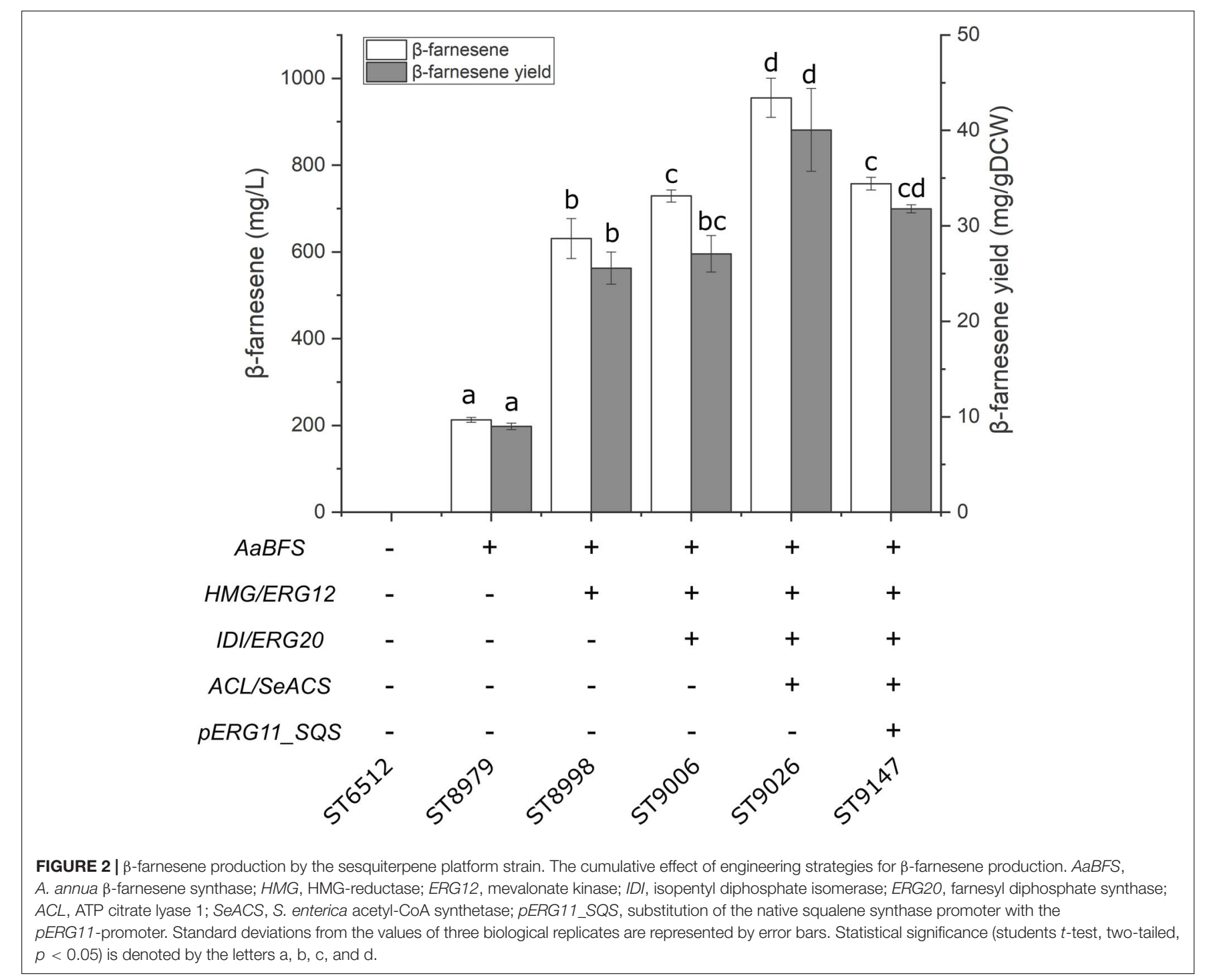

and ST9010, respectively. The lanosterol synthase catalyzes the committed step toward sterols from 2,3-oxidosqualene and limiting this step could potentially increase the pool of 2,3-oxidosqualene and squalene. Building on ST9010, ACL and SeACS were expressed and subsequently, IDI and ERG20 were overexpressed. Further overexpression of the native squalene synthase $(S Q S)$ resulted in squalene platform strain which produced $402.4 \pm 90 \mathrm{mg} / \mathrm{L}$ squalene. When instead $S Q E$ and SQS were co-overexpressed generating the complex triterpenoid platform strain (ST9106), only $262.7 \pm 5.2 \mathrm{mg} / \mathrm{L}$ squalene were produced, while levels of $22 \pm 5.9 \mathrm{mg} / \mathrm{L} \mathrm{2,3-oxidosqualene}$ was detected. Indeed, 2,3-oxidosqualene was measured for all the squalene producing strains but could only be detected in the complex triterpenoid platform strain. Overexpression of the native diacylglycerol O-acyltransferase (DGA1) together in the squalene platform strain resulted in $320 \pm 16.2 \mathrm{mg} / \mathrm{L}$ squalene, less than when only expressing SQS. Overexpression of the native DGA1 in combination with a truncated version of $H M G$ in $S$. cerevisiae increased squalene titers, probably due to an increased lipid content providing storage for squalene accumulation (Wei et al., 2018). However, overexpression of DGA1 in Y. lipolytica did not benefit squalene production in the context of this study, likely due to the diversion of acetyl-CoA to lipid biogenesis (Figure 1).

\section{Diterpene and Carotenoid Platform Strain}

To engineer a platform strain for the production of diterpenoids and carotenoids, the genes HMG, ERG12, ACL, SeACS, and IDI were overexpressed, while the native squalene promoter was swapped with the relatively weak promoter $p E R G 11$ to reduce the flux toward squalene ( $P E R G 11 \_S Q S$ ). Additionally, either GGPPS or ERG20 $0^{F 8 C}$ was overexpressed to generate the diterpenoid/carotenoid platform strains ST9150 and ST9203, respectively. To test the potential of these platform strains, the genes encoding phytoene desaturase (XdCtrl) and bifunctional phytoene synthase/lycopene cyclase $(X d C \operatorname{tr} Y B)$ from 


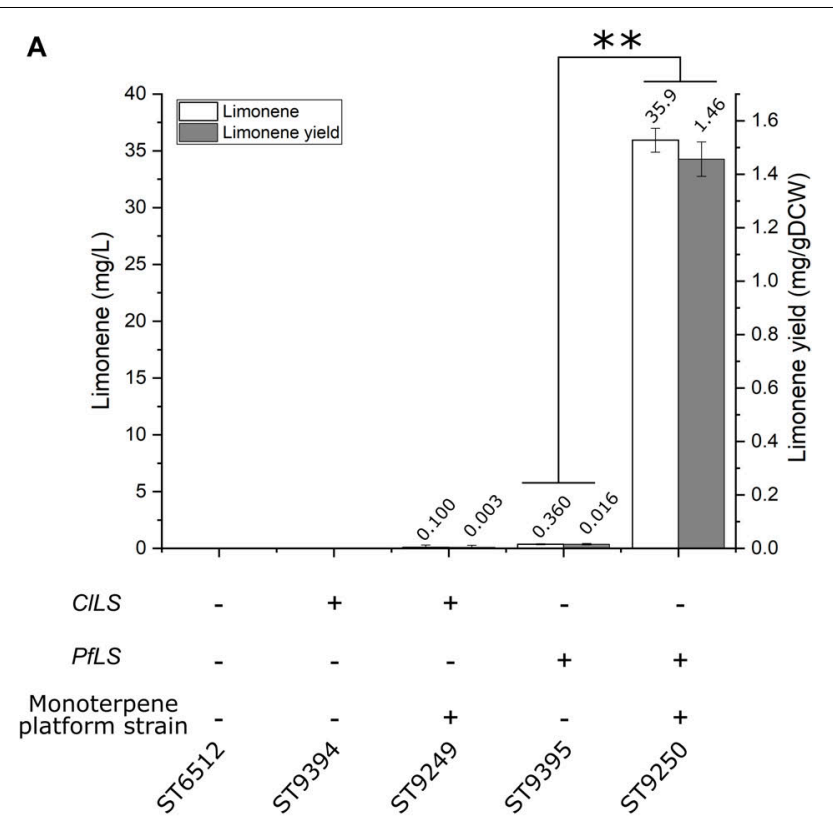

B

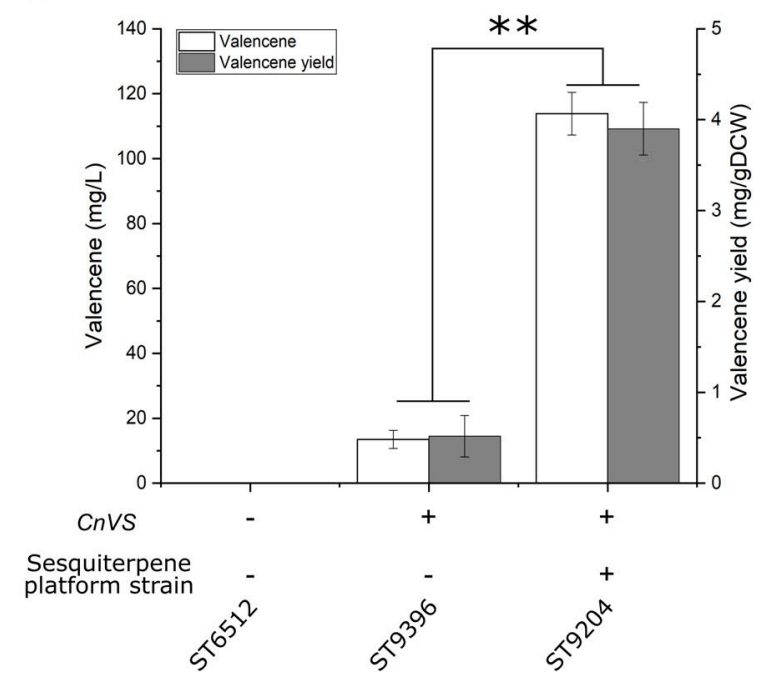

FIGURE 3 | Limonene and valencene production by platform strains. (A) Limonene production in base and monoterpene platform strain. CILS, C. Iemon limonene synthase; PfLS, P. frutescens limonene synthase. The monoterpene platform strain contains the following gene overexpressions of HMG, ERG12, ACL, SeACS, IDI, ERG2OF88W-N119W, and swapping the native squalene promoter (DERG11_SQS). (B) Valencene production in base and sesquiterpene platform strain. CnVS, C. nootkatensis valencene synthase. The sesquiterpene platform strain contains the same modifications as the monoterpene platform strain, with the exception of ERG20 instead of $E R G 2 O^{F 88 W-N 119 W}$. Standard deviations from the values of three biological replicates are represented by error bars. Double asterisks marks significant change (student's $t$-test, two-tailed, $p<0.001$ ).

$X$. dendrorhous for the production of $\beta$-carotene were expressed in both strains, generating ST9251 and ST9253, respectively. ST9251 produced $158 \pm 24.1 \mathrm{mg} / \mathrm{L} \beta$-carotene, while ST9253 produced $164 \pm 37.6 \mathrm{mg} / \mathrm{L} \beta$-carotene (Figure 5).

\section{DISCUSSION}

This study displays the capability of the oleaginous yeast $Y$. lipolytica for terpenoid production by the construction of tailored terpene platform strains. Each platform strain was designed for increased production of either mono-, sesqui-, tri-, or diterpenoids and carotenoids, and production potential was tested with terpenoids from the appropriate class. The effects of four different metabolic engineering strategies for sesquiterpenoid production were investigated in a strain expressing $A a B F S$ for $\beta$-farnesene. Overexpression of $H M G$ and ERG12 had previously been shown to increase limonene production 112-fold to $23.56 \mathrm{mg} / \mathrm{L}$ in $Y$. lipolytica (Cao et al., 2016). The strategy of $H M G$-overexpression is very commonly used for terpenoid production in Y. lipolytica, with both truncated and non-truncated versions, and variants from other species being utilized, while the ERG12 gene often is overexpressed together with the whole MVA-pathway (Table 1). Indeed, overexpression $E R G 12$ and $H M G$ increased $\beta$-farnesene production approximately 3-fold, from $212.7 \pm 5.6 \mathrm{mg} / \mathrm{L}$ to $631 \pm 46.1 \mathrm{mg} / \mathrm{L}$, demonstrating that this strategy works well for $\beta$-farnesene production. By further overexpression of ERG20, IDI, SeACS, and ACL1 the titer of $955 \pm 45.1 \mathrm{mg} / \mathrm{L}$ $\beta$-farnesene was reached. Improvement of acetyl-CoA by overexpression of SeACS and ACL1 had a favorable effect on $\beta$-farnesene production which is consistent with previous reports demonstrating a similar effect for squalene production in Y. lipolytica (Huang et al., 2018). However, recent studies have found that strategies that increase $\beta$-oxidation such as overexpression of multifunctional $\beta$-oxidation enzyme 1 (MFE1) or implementing alternative routes for acetyl-CoA biogenesis by expression of phosphoketolases $(P K)$ and phosphotransacetylases $(P T A)$, the non-glycolytic oxidation pathway, can also increase terpenoid titers (Table 1; Jin et al., 2019; Lu et al., 2020). It would be highly interesting for future research to compare these strategies for terpenoid production in a systematic manner. $\beta$-farnesene has been produced at titers of $130 \mathrm{~g} / \mathrm{L}$ by highly modified S. cerevisiae in 200,000-liter bioreactors and therefore is an excellent terpene to demonstrate the effect of metabolic engineering strategies (Meadows et al., 2016). While $\beta$-farnesene has not been produced previously in $Y$. lipolytica, the isomer $\alpha$-farnesene has been produced by engineered $Y$. lipolytica at $25.55 \mathrm{~g} / \mathrm{L}$ in 1 -liter bioreactors (Liu et al., 2019). Interestingly, switching the endogenous squalene promoter with the weak $p E R G 11$ promoter did not benefit $\beta$-farnesene production, although the same strategy was shown to boost $\beta$-carotene production in $Y$. lipolytica (Kildegaard et al., 2017). This could be caused the combination of reduced cellular sterol-content and high $\beta$-farnesene levels that may alter the lipid membrane properties and induce toxicity. It has been demonstrated that several low-molecularweight terpenoids like limonene, geraniol, and pinene can be toxic to S. cerevisiae (Uribe et al., 1985; Liu et al., 2013; Zhao et al., 2016). Indeed, pinene was shown to potentially increase lipid membrane fluidity, while limonene could adversely affect the yeast cell wall (Brennan et al., 2013). Candida albicans cells exposed to high doses of linalool or 


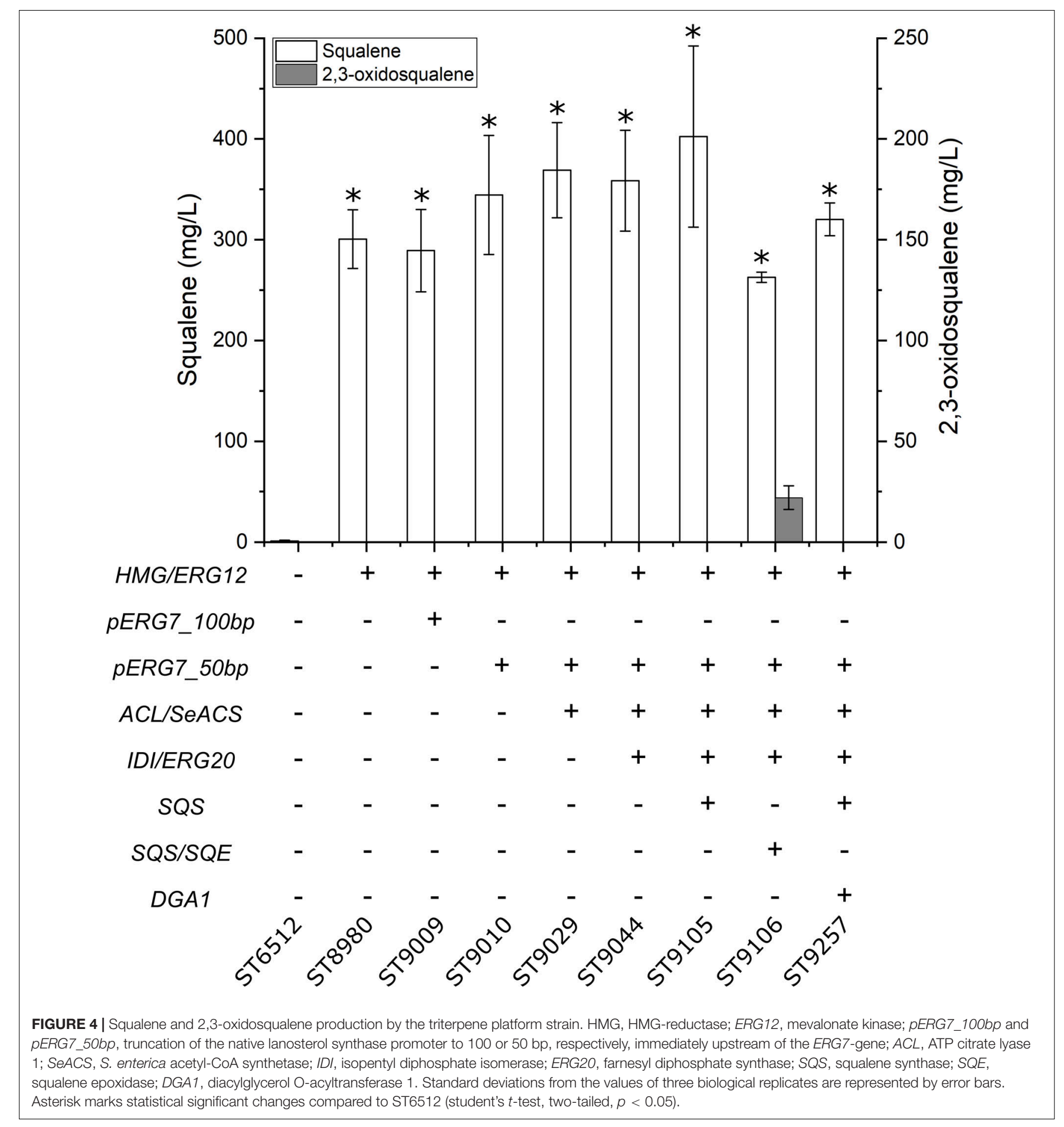

its derivative linalyl acetate displayed apoptotic phenotypes and highly fluidized membranes leading to cell death (Khan et al., 2014; Blaskó et al., 2017). Two-phase cultivation with an organic phase has been shown to alleviate the toxicity of small terpenoids against $S$. cerevisiae (Brennan et al., 2012). Indeed, a dodecane phase can be used to reduce cell toxicity and to accumulate the produced compounds (Tippmann et al., 2016). It is therefore likely that the utilization of a dodecane phase for the accumulation of limonene, valencene, and $\beta$-farnesene also helped to limit adverse effects in Y. lipolytica in this study. The sesquiterpene valencene is the precursor of nootkatone, a compound used for cosmetics and fragrances, with insect repellant properties (Leonhardt and Berger, 2014). By simply integrating CnVS in the optimized sesquiterpene platform strain, $113.9 \pm 6.6 \mathrm{mg} / \mathrm{L}$ valencene was produced, an approximately 8.4-fold increase 


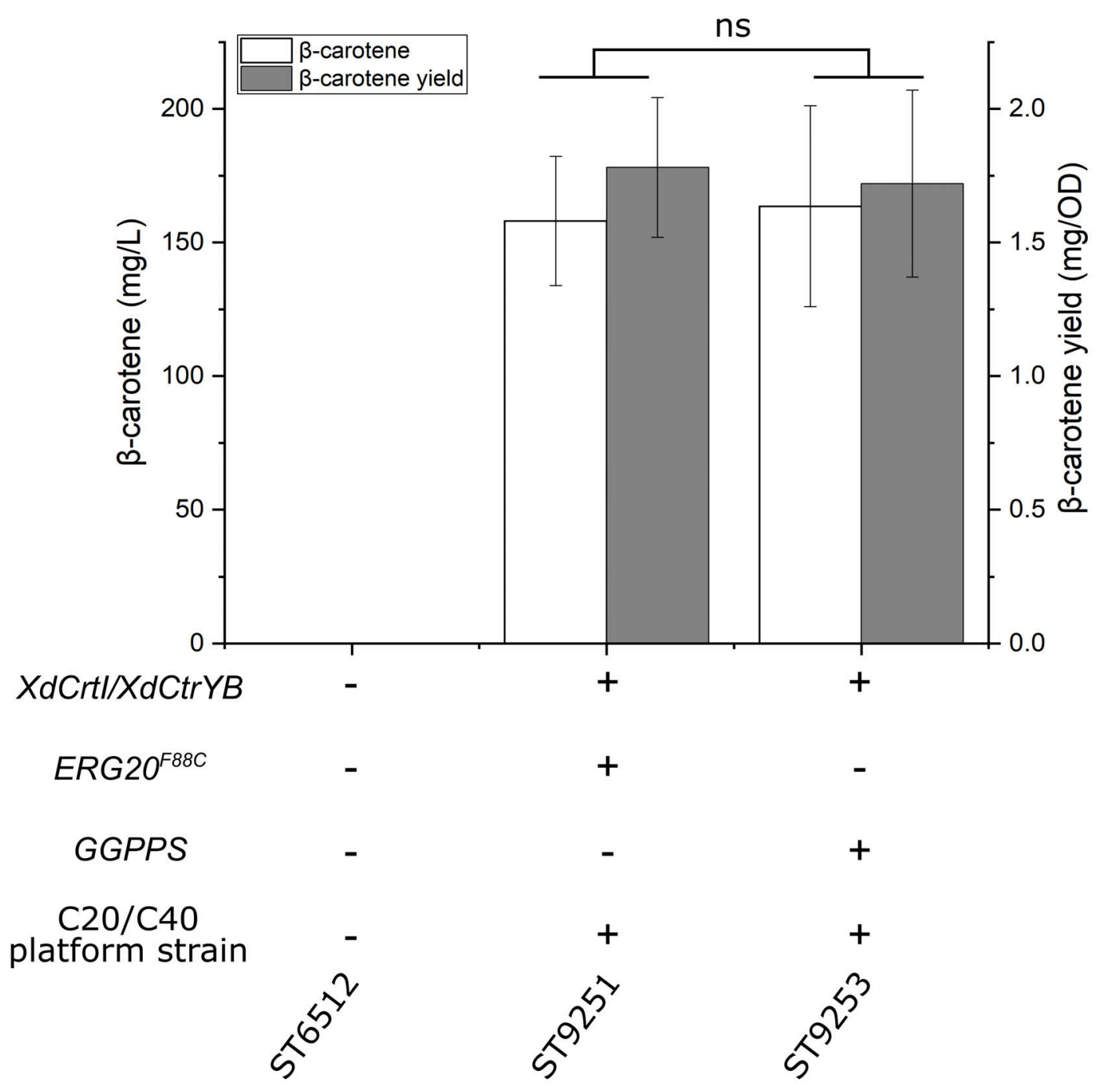

FIGURE 5 | $\beta$-carotene production by the diterpene/carotenoid platform strains. XdCrtl, X. dendrorhous phytoene desaturase; XdCrtYB, X. dendrorhous bi-functional phytoene synthase/lycopene cyclase; ERG2OF88C, mutated version with geranylgeranyl diphosphate synthase activity; GGPPS, geranylgeranyl diphosphate synthase. The diterpene/carotenoid (C20/C40) platform strain contains the following gene overexpressions HMG, ERG12, ACL, SeACS, and IDI, while the native squalene promoter was swapped ( $\left.D E R G 11 \_S Q S\right)$. Average values and standard deviations for each strain are based on two individual clones with three biological replicates each. Statistical non-significance is marked with ns (student's $t$-test, two-tailed, $p>0.05$ ).

over the base strain. This example clearly illustrates the usefulness of pre-engineered strains for achieving high terpene titers quickly and without post-optimization of the strain. By comparison, previous valence production as precursor for nootkatone in engineered $Y$. lipolytica resulted in $22.8 \mathrm{mg} / \mathrm{L}$ valencene and $978.2 \mu \mathrm{g} / \mathrm{L}$ nootkatone during shake flask cultivation (Guo et al., 2018). The sesquiterpene platform strain is a powerful platform for the production of terpenes like valencene and could be engineered to produce more complex sesquiterpenoids. Limonene has been produced at $23.56 \mathrm{mg} / \mathrm{L}$ and $1.36 \mathrm{mg} / \mathrm{gDCW}$ in shake flasks by the $Y$. lipolytica strain Pof1-LN-051 expressing a limonene synthase from Korean mint Agastache rugosa (Cao et al., 2016). In a subsequent report, an additional limonene synthase copy was expressed in Pof1LN-051, where after cultivation in $1.5 \mathrm{~L}$ bioreactors resulted in $165.3 \mathrm{mg} / \mathrm{L}$, which is the highest reported limonene titer for Y. lipolytica (Cheng et al., 2019). By comparison, the expression of PfLS in the monoterpenoid platform strain resulted in $35.9 \pm 1.1 \mathrm{mg} / \mathrm{L}$ and $1.46 \pm 0.064 \mathrm{mg} / \mathrm{gDCW}$ limonene in small-scale cultivation. Therefore, the monoterpene platform strain clearly holds a great potential for limonene production, which potentially could be improved substantially by multicopy integration of PfLS and scale-up into bioreactors. By integrating $H M G, E R G 12, E R G 20, I D I, S Q S$, and truncating the native lanosterol promoter, a strain for squalene production was constructed. This strain was able to produce $402.4 \pm 90 \mathrm{mg} / \mathrm{L}$ squalene by small-scale cultivation. Other reports of squalene production in highly modified $Y$. lipolytica show titers up to $531.6 \mathrm{mg} / \mathrm{L}$ by a strain with at least twelve modifications relevant to squalene production, including overexpression of the 
entire MVA-pathway and knockouts of peroxisomal $\beta$-oxidation (pox) genes which could affect acetyl-CoA balance (Table 1; Gao et al., 2017). By comparison the squalene platform strain from this study contained only seven gene overexpression and one gene downregulation. Therefore, further engineering and scale-up could potentially improve the production of squalene described herein. Overexpression of $S Q E$ in combination with the modifications described for the squalene production strain resulted in $22 \pm 5.9 \mathrm{mg} / \mathrm{L} \mathrm{2,3-oxidosqualene.} \mathrm{This} \mathrm{platform}$ strain can potentially be used for the production of complex triterpenoids. Since $S Q E$ was expressed under the $P G P D$ promoter, expressing additional copies of $S Q E$, potentially under the relatively stronger pTEFintron-promoter, could raise 2,3oxidosqualene levels further (Holkenbrink et al., 2018). The production of $\beta$-carotene for the carotenoid platform strains, $158 \pm 24.1 \mathrm{mg} / \mathrm{L}$ or $164 \pm 37.6 \mathrm{mg} / \mathrm{L}$ for ST9251 or ST9253, respectively, are lower than in previous reports (Kildegaard et al., 2017; Larroude et al., 2018). To achieve $6.5 \mathrm{~g} / \mathrm{L} \beta$-carotene, three copies of the pathway enzymes (carB and CarRP) and GGPPS were integrated in a W29-derived strain engineered for high lipogenesis (Larroude et al., 2018). Therefore, further engineering of the downstream biosynthetic pathway may be necessary when using the platform strains.

\section{CONCLUSION}

Tailored platform strains for the production of mono-, sesqui-, tri-, or diterpenes and carotenoids were constructed, and their potential was tested by the production of limonene, $\beta$-farnesene and valencene, squalene and 2,3-oxidosqualene, and $\beta$-carotene, respectively. These improved platform strains were metabolically engineered for improved terpenoid production and their use could result in almost 100 -fold improvement production for some terpenes compared to base strains. These platform strains can potentially be improved even further by engineering and scale-up into bioreactors.

\section{STRAIN AVAILABILITY STATEMENT}

The platform strains and representative terpenoid producing strains from this study can be requested from Euroscarf. The Euroscarf accession numbers are given in parenthesis. ST9202, monoterpene platform strain (Y41400). ST9250, monoterpene platform strain with PfLS (Y41401). ST9395, base strain with PfLS (Y41402). ST9149, sesquiterpene platform

\section{REFERENCES}

Angerer, H., Radermacher, M., Ma kowska, M., Steger, M., Zwicker, K., Heide, H., et al. (2014). The LYR protein subunit NB4M/NDUFA6 of mitochondrial complex I anchors an acyl carrier protein and is essential for catalytic activity. Proc. Natl. Acad. Sci. U.S.A. 111, 5207-5212. doi: 10.1073/pnas.132243 8111

Ashour, M., Wink, M., and Gershenzon, J. (2010). "Biochemistry of terpenoids: monoterpenes, sesquiterpenes and diterpenes," in Biochemistry of Plant strain (Y41403). ST9204, sesquiterpene platform strain with CnVS (Y41404). ST9396, base strain with CnVS (Y41405). ST9105, squalene production strain (Y41406). ST9106, triterpene platform strain (Y41407). ST6512, base strain (Y41408). ST9203, diterpene/carotenoid platform strain with ERG20 ${ }^{F 8 C}$ (Y41409). ST9150, diterpene/carotenoid platform strain with GGPPS (Y41410). The $\beta$-farnesene test-strains ST8979, ST8998, ST9006, ST9026, and ST9147 (Y41411-15).

\section{DATA AVAILABILITY STATEMENT}

The platform strains and representative strains for terpenoid production can be requested from Euroscarf or the authors.

\section{AUTHOR CONTRIBUTIONS}

JA, KK, and IB designed the experiments. MC, SJ, and JA carried out the experiments. $\mathrm{MK}$ provided critical assistance in developing GC-FID and HP-LC methods. JA analyzed the data. JA and IB wrote the manuscript. All authors contributed to the article and approved the submitted version.

\section{FUNDING}

The research was funded by The Novo Nordisk Foundation (Grant agreements NNF15OC0016592 and NNF10CC1016517). IB and KK acknowledge the financial support from the European Union's Horizon 2020 Research and Innovation Programs (European Research Council, YEAST-TRANS project no. 757384 and OLEFINE project no. 760798).

\section{ACKNOWLEDGMENTS}

The authors thank the ARS Culture Collection, NCAUR, United States, for providing the W29 Y. lipolytica strain Y-63764 and Volker Zickerman for providing the Y. lipolytica strain GB20 (Angerer et al., 2014).

\section{SUPPLEMENTARY MATERIAL}

The Supplementary Material for this article can be found online at: https://www.frontiersin.org/articles/10.3389/fbioe. 2020.00945/full\#supplementary-material

Secondary Metabolism, ed. M. Wink (Oxford: Wiley-Blackwell), 258-303. doi: 10.1002/9781444320503.ch5

Beekwilder, J., van Houwelingen, A., Cankar, K., van Dijk, A. D. J., de Jong, R. M., Stoopen, G., et al. (2014). Valencene synthase from the heartwood of Nootka cypress (Callitropsis nootkatensis) for biotechnological production of valencene. Plant Biotechnol. J. 12, 174-182. doi: 10.1111/pbi.12124

Benchling (2020). Cloud-Based Informatics Platform for Life Sciences R\&D | Benchling. Available online at: https://www.benchling.com/ (accessed March 9, 2020). 
Blaskó, Á, Gazdag, Z., Gróf, P., Máté, G., Sárosi, S., Krisch, J., et al. (2017). Effects of clary sage oil and its main components, linalool and linalyl acetate, on the plasma membrane of Candida albicans: an in vivo EPR study. Apoptosis 22, 175-187. doi: 10.1007/s10495-016-1321-7

Brennan, T. C. R., Krömer, J. O., and Nielsen, L. K. (2013). Physiological and Transcriptional Responses of Saccharomyces cerevisiae to d-Limonene show changes to the cell wall but not to the plasma membrane. Appl. Environ. Microbiol. 79, 3590-3600. doi: 10.1128/AEM.00463-13

Brennan, T. C. R., Turner, C. D., Krömer, J. O., and Nielsen, L. K. (2012). Alleviating monoterpene toxicity using a two-phase extractive fermentation for the bioproduction of jet fuel mixtures in Saccharomyces cerevisiae. Biotechnol. Bioeng. 109, 2513-2522. doi: 10.1002/bit.24536

Bröker, J. N., Müller, B., van Deenen, N., Prüfer, D., and Schulze Gronover, C. (2018). Upregulating the mevalonate pathway and repressing sterol synthesis in Saccharomyces cerevisiae enhances the production of triterpenes. Appl. Microbiol. Biotechnol. 102, 6923-6934. doi: 10.1007/s00253-018-9154-7

Brown, N. P., Leroy, C., and Sander, C. (1998). MView: a web-compatible database search or multiple alignment viewer. Bioinformatics 14, 380-381. doi: 10.1093/ bioinformatics/14.4.380

Cao, X., Lv, Y. B., Chen, J., Imanaka, T., Wei, L. J., and Hua, Q. (2016). Metabolic engineering of oleaginous yeast Yarrowia lipolytica for limonene overproduction. Biotechnol. Biofuels 9, 1-11. doi: 10.1186/s13068-016-0626-7

Cao, X., Wei, L. J., Lin, J. Y., and Hua, Q. (2017). Enhancing linalool production by engineering oleaginous yeast Yarrowia lipolytica. Bioresour. Technol. 245, 1641-1644. doi: 10.1016/j.biortech.2017.06.105

Cheng, B.-Q., Wei, L.-J., Lv, Y.-B., Chen, J., and Hua, Q. (2019). Elevating limonene production in oleaginous Yeast Yarrowia lipolytica via genetic engineering of limonene biosynthesis pathway and optimization of medium composition. Biotechnol. Bioprocess Eng. 24, 500-506. doi: 10.1007/s12257-018-0497-9

Christen, S., and Sauer, U. (2011). Intracellular characterization of aerobic glucose metabolism in seven yeast species by $13 \mathrm{C}$ flux analysis and metabolomics. FEMS Yeast Res. 11, 263-272. doi: 10.1111/j.1567-1364.2010.00713.x

Cravens, A., Payne, J., and Smolke, C. D. (2019). Synthetic biology strategies for microbial biosynthesis of plant natural products. Nat. Commun. 10, 1-12. doi: 10.1038/s41467-019-09848-w

Czajka, J. J., Nathenson, J. A., Benites, V. T., Baidoo, E. E. K., Cheng, Q., Wang, Y., et al. (2018). Engineering the oleaginous yeast Yarrowia lipolytica to produce the aroma compound $\beta$-ionone. Microb. Cell Fact. 17, 1-13. doi: 10.1186/s12934018-0984-x

Du, H. X., Xiao, W. H., Wang, Y., Zhou, X., Zhang, Y., Liu, D., et al. (2016). Engineering Yarrowia lipolytica for campesterol overproduction. PLoS One 11:e0146773. doi: 10.1371/journal.pone.0146773

Dujon, B., Sherman, D., Fischer, G., Durrens, P., Casaregola, S., Lafontaine, I., et al. (2004). Genome evolution in yeasts. Nature 430, 35-44. doi: 10.1038/ nature 02579

Evolva (2020). Our Valencene the Natural Choice for Flavour and Fragrance Applications. Available online at: https://evolva.com/evevalencene/ (accessed March 9, 2020).

Fernandes, S. R., Barreiros, L., Oliveira, R. F., Cruz, A., Prudêncio, C., Oliveira, A. I., et al. (2019). Chemistry, bioactivities, extraction and analysis of azadirachtin: state-of-the-art. Fitoterapia 134, 141-150. doi: 10.1016/j.fitote.2019.02.006

Gao, S., Tong, Y., Zhu, L., Ge, M., Zhang, Y., Chen, D., et al. (2017). Iterative integration of multiple-copy pathway genes in Yarrowia lipolytica for heterologous $\beta$-carotene production. Metab. Eng. 41, 192-201. doi: 10.1016/j. ymben.2017.04.004

George, K. W., Alonso-Gutierrez, J., Keasling, J. D., and Lee, T. S. (2015). "Isoprenoid drugs, biofuels, and Chemicals-Artemisinin, farnesene, and beyond," in Advances in Biochemical Engineering/Biotechnology, eds J. Schrader and J. Bohlmann (Cham: Springer), 355-389. doi: 10.1007/10_2014_288

Groenewald, M., Boekhout, T., Neuvéglise, C., Gaillardin, C., Van Dijck, P. W. M., and Wyss, M. (2014). Yarrowia lipolytica: safety assessment of an oleaginous yeast with a great industrial potential. Crit. Rev. Microbiol. 40, 187-206. doi: 10.3109/1040841X.2013.770386

Guo, X., Sun, J., Li, D., and Lu, W. (2018). Heterologous biosynthesis of (+)nootkatone in unconventional yeast Yarrowia lipolytica. Biochem. Eng. J. 137, 125-131. doi: 10.1016/j.bej.2018.05.023

Han, J. Y., Seo, S. H., Song, J. M., Lee, H., and Choi, E. S. (2018). High-level recombinant production of squalene using selected Saccharomyces cerevisiae strains. J. Ind. Microbiol. Biotechnol. 45, 239-251. doi: 10.1007/s10295-0182018-4

Hill, R. A., and Connolly, J. D. (2017). Triterpenoids. Nat. Prod. Rep. 34, 90-122. doi: 10.1039/C6NP00094K

Holkenbrink, C., Dam, M. I., Kildegaard, K. R., Beder, J., Dahlin, J., Doménech Belda, D., et al. (2018). EasyCloneYALI: CRISPR/Cas9-based synthetic toolbox for engineering of the yeast Yarrowia lipolytica. Biotechnol. J. 13:1700543. doi: 10.1002/biot.201700543

Huang, Y.-Y., Jian, X.-X., Lv, Y.-B., Nian, K.-Q., Gao, Q., Chen, J., et al. (2018). Enhanced squalene biosynthesis in Yarrowia lipolytica based on metabolically engineered acetyl-CoA metabolism. J. Biotechnol. 281, 106-114. doi: 10.1016/j. jbiotec.2018.07.001

Ignea, C., Pontini, M., Maffei, M. E., Makris, A. M., and Kampranis, S. C. (2014). Engineering monoterpene production in yeast using a synthetic dominant negative geranyl diphosphate synthase. ACS Synth. Biol. 3, 298-306. doi: 10. $1021 /$ sb400115e

Ignea, C., Trikka, F. A., Nikolaidis, A. K., Georgantea, P., Ioannou, E., Loupassaki, S., et al. (2015). Efficient diterpene production in yeast by engineering Erg20p into a geranylgeranyl diphosphate synthase. Metab. Eng. 27, 65-75. doi: 10. 1016/j.ymben.2014.10.008

Jia, D., Xu, S., Sun, J., Zhang, C., Li, D., and Lu, W. (2019). Yarrowia lipolytica construction for heterologous synthesis of $\alpha$-santalene and fermentation optimization. Appl. Microbiol. Biotechnol. 103, 3511-3520. doi: 10.1007/s00253019-09735-w

Jin, C.-C., Zhang, J.-L., Song, H., and Cao, Y.-X. (2019). Boosting the biosynthesis of betulinic acid and related triterpenoids in Yarrowia lipolytica via multimodular metabolic engineering. Microb. Cell Fact. 18:77. doi: 10.1186/ s12934-019-1127-8

Janocha, S., Schmitz, D., and Bernhardt, R. (2015). Terpene hydroxylation with microbial cytochrome P450 monooxygenases. Adv. Biochem. Eng. Biotechnol. 148, 215-250. doi: 10.1007/10_2014_296

Khan, A., Ahmad, A., Khan, L. A., and Manzoor, N. (2014). Ocimum sanctum (L.) essential oil and its lead molecules induce apoptosis in Candida albicans. Res. Microbiol. 165, 411-419. doi: 10.1016/j.resmic.2014.05.031

Kildegaard, K. R., Adiego-Pérez, B., Doménech Belda, D., Khangura, J. K., Holkenbrink, C., and Borodina, I. (2017). Engineering of Yarrowia lipolytica for production of astaxanthin. Synth. Syst. Biotechnol. 2, 287-294. doi: 10.1016/ j.synbio.2017.10.002

Larroude, M., Celinska, E., Back, A., Thomas, S., Nicaud, J. M., and Ledesma-Amaro, R. (2018). A synthetic biology approach to transform Yarrowia lipolytica into a competitive biotechnological producer of $\beta$-carotene. Biotechnol. Bioeng. 115, 464-472. doi: 10.1002/bit.26473

Leonhardt, R.-H., and Berger R. G. (2014). "Nootkatone," in Biotechnology of Isoprenoids, Vol. 148, Advances in Biochemical Engineering/Biotechnology, eds J. Schrader and J. Bohlmann (Berlin: Springer). doi: 10.1007/10_2014_279

Li, D., Wu, Y., Wei, P., Gao, X., Li, M., Zhang, C., et al. (2020). Metabolic engineering of Yarrowia lipolytica for heterologous oleanolic acid production. Chem. Eng. Sci. 218:115529. doi: 10.1016/j.ces.2020.115529

Li, D., Wu, Y., Zhang, C., Sun, J., Zhou, Z., and Lu, W. (2019). Production of triterpene ginsenoside compound $\mathrm{K}$ in the non-conventional yeast Yarrowia lipolytica. J. Agric. Food Chem. 67, 2581-2588. doi: 10.1021/acs.jafc. $9 \mathrm{~b} 00009$

Liu, J., Zhu, Y., Du, G., Zhou, J., and Chen, J. (2013). Response of Saccharomyces cerevisiae to D-limonene-induced oxidative stress. Appl. Microbiol. Biotechnol. 97, 6467-6475. doi: 10.1007/s00253-013-4931-9

Liu, Y., Jiang, X., Cui, Z., Wang, Z., Qi, Q., and Hou, J. (2019). Engineering the oleaginous yeast Yarrowia lipolytica for production of $\alpha$-farnesene. Biotechnol. Biofuels 12:296. doi: 10.1186/s13068-019-1636-Z

Lu, Y., Yang, Q., Lin, Z., and Yang, X. (2020). A modular pathway engineering strategy for the high-level production of $\beta$-ionone in Yarrowia lipolytica. Microb. Cell Fact. 19:49. doi: 10.1186/s12934-020-01309-0

Marella, E. R., Dahlin, J., Dam, M. I., ter Horst, J., Christensen, H. B., Sudarsan, S., et al. (2019). A single-host fermentation process for the production of flavor lactones from non-hydroxylated fatty acids. Metab. Eng S1096-7176:30254-X. doi: 10.1016/j.ymben.2019.08.009

Marsafari, M., and Xu, P. (2020). Debottlenecking mevalonate pathway for antimalarial drug precursor amorphadiene biosynthesis in Yarrowia lipolytica. Metab. Eng. Commun. 10:e00121. doi: 10.1016/j.mec.2019.e00121 
Matthäus, F., Ketelhot, M., Gatter, M., and Barth, G. (2014). Production of lycopene in the non-carotenoid-producing yeast Yarrowia lipolytica. Appl. Environ. Microbiol. 80, 1660-1669. doi: 10.1128/AEM.03167-13

Meadows, A. L., Hawkins, K. M., Tsegaye, Y., Antipov, E., Kim, Y., Raetz, L., et al. (2016). Rewriting yeast central carbon metabolism for industrial isoprenoid production. Nature 537, 694-697. doi: 10.1038/nature19769

Moser, S., and Pichler, H. (2019). Identifying and engineering the ideal microbial terpenoid production host. Appl. Microbiol. Biotechnol. 103, 5501-5516. doi: 10.1007/s00253-019-09892-y

Pang, Y., Zhao, Y., Li, S., Zhao, Y., Li, J., Hu, Z., et al. (2019). Engineering the oleaginous yeast Yarrowia lipolytica to produce limonene from waste cooking oil. Biotechnol. Biofuels 12, 1-18. doi: 10.1186/s13068-019-1580-y

Schwartz, C., Frogue, K., Misa, J., and Wheeldon, I. (2017). Host and pathway engineering for enhanced lycopene biosynthesis in Yarrowia lipolytica. Front. Microbiol. 8:2233. doi: 10.3389/fmicb.2017.02233

Sun, J., Zhang, C., Nan, W., Li, D., Ke, D., and Lu, W. (2019). Glycerol improves heterologous biosynthesis of betulinic acid in engineered Yarrowia lipolytica. Chem. Eng. Sci. 196, 82-90. doi: 10.1016/j.ces.2018.10.052

Tetali, S. D. (2018). Terpenes and isoprenoids: a wealth of compounds for global use. Planta 249, 1-8. doi: 10.1007/s00425-018-3056-x

Tippmann, S., Nielsen, J., and Khoomrung, S. (2016). Improved quantification of farnesene during microbial production from Saccharomyces cerevisiae in twoliquid-phase fermentations. Talanta 146, 100-106. doi: 10.1016/j.talanta.2015. 08.031

Tramontin, L. R. R., Kildegaard, K. R., Sudarsan, S., and Borodina, I. (2019). Enhancement of astaxanthin biosynthesis in oleaginous yeast Yarrowia lipolytica via microalgal pathway. Microorganisms 7:472. doi: 10.3390/ microorganisms7100472

Turck, D., Castenmiller, J., de Henauw, S., Hirsch-Ernst, K., Kearney, J., Maciuk, A., et al. (2019). Safety of Yarrowia lipolytica yeast biomass as a novel food pursuant to Regulation (EU) 2015/2283. EFSA J. 17:e05594. doi: 10.2903/j.efsa.2019.5594

Uribe, S., Ramirez, J., and Pena, A. (1985). Effects of $\beta$-Pinene on yeast membrane functions. J. Bacteriol 161, 1195-1200. doi: 10.1128/jb.161.3.1195-1200.1985

Wei, L. J., Kwak, S., Liu, J. J., Lane, S., Hua, Q., Kweon, D. H., et al. (2018). Improved squalene production through increasing lipid contents in Saccharomyces cerevisiae. Biotechnol. Bioeng. 115, 1793-1800. doi: 10.1002/bit.26595
Wu, Y., Xu, S., Gao, X., Li, M., Li, D., and Lu, W. (2019). Enhanced protopanaxadiol production from xylose by engineered Yarrowia lipolytica. Microb. Cell Fact. 18, 1-12. doi: 10.1186/s12934-019-1136-7

Xu, W., Ma, X., and Wang, Y. (2016). Production of squalene by microbes: an update. World J. Microbiol. Biotechnol. 32:195. doi: 10.1007/s11274-016-2155-8

Yang, X., Nambou, K., Wei, L., and Hua, Q. (2016). Heterologous production of $\alpha$ farnesene in metabolically engineered strains of Yarrowia lipolytica. Bioresour. Technol. 216, 1040-1048. doi: 10.1016/j.biortech.2016.06.028

Zhang, X.-K., Wang, D.-N., Chen, J., Liu, Z.-J., Wei, L.-J., and Hua, Q. (2020). Metabolic engineering of $\beta$-carotene biosynthesis in Yarrowia lipolytica. Biotechnol. Lett. 42, 945-956. doi: 10.1007/s10529-020-02844-x

Zhang, Y., Nielsen, J., and Liu, Z. (2017a). Engineering yeast metabolism for production of terpenoids for use as perfume ingredients, pharmaceuticals and biofuels. FEMS Yeast Res. 17, 1-11. doi: 10.1093/femsyr/fox080

Zhang, Y., Wang, Y., Yao, M., Liu, H., Zhou, X., Xiao, W., et al. (2017b). Improved campesterol production in engineered Yarrowia lipolytica strains. Biotechnol. Lett. 39, 1033-1039. doi: 10.1007/s10529-017-2331-4

Zhao, J., Bao, X., Li, C., Shen, Y., and Hou, J. (2016). Improving monoterpene geraniol production through geranyl diphosphate synthesis regulation in Saccharomyces cerevisiae. Appl. Microbiol. Biotechnol. 100, 4561-4571. doi: 10. 1007/s00253-016-7375-1

Zyad, A., Tilaoui, M., Jaafari, A., Oukerrou, M. A., and Mouse, H. A. (2018). More insights into the pharmacological effects of artemisinin. Phyther. Res. 32, 216-229. doi: 10.1002/ptr.5958

Conflict of Interest: The authors declare that the research was conducted in the absence of any commercial or financial relationships that could be construed as a potential conflict of interest.

Copyright (c) 2020 Arnesen, Kildegaard, Cernuda Pastor, Jayachandran, Kristensen and Borodina. This is an open-access article distributed under the terms of the Creative Commons Attribution License (CC BY). The use, distribution or reproduction in other forums is permitted, provided the original author(s) and the copyright owner(s) are credited and that the original publication in this journal is cited, in accordance with accepted academic practice. No use, distribution or reproduction is permitted which does not comply with these terms. 